\title{
La littératie, entre cognitivisme et culturalisme
}

Vers une approche multimodale et « reconnectée » de la littératie scolaire?

\section{Christophe Joigneaux}

\section{(2) OpenEdition}

12 Journals

Édition électronique

URL : http://journals.openedition.org/pratiques/7042

DOI : $10.4000 /$ pratiques.7042

ISSN : 2425-2042

Éditeur

Centre de recherche sur les médiations (CREM)

Référence électronique

Christophe Joigneaux, «La littératie, entre cognitivisme et culturalisme», Pratiques [En ligne], 183-184 | 2019, mis en ligne le 30 décembre 2019, consulté le 09 octobre 2020. URL : http://

journals.openedition.org/pratiques/7042 ; DOI : https://doi.org/10.4000/pratiques.7042

Ce document a été généré automatiquement le 9 octobre 2020.

(c) Tous droits réservés 


\section{La littératie, entre cognitivisme et culturalisme}

Vers une approche multimodale et « reconnectée » de la littératie scolaire?

\section{Christophe Joigneaux}

1 Étudier les pratiques d'«entrée dans l'écrit» ou "dans la culture de l'écrit», pour reprendre des expressions en usage dans les programmes de l'école maternelle française (Ministère de l'Éducation nationale et de la Jeunesse, 2015, p. 7), finit par questionner ce que l'on entend communément par «écrit» ou par "littératie » (Laparra \& Margolinas, 2016). En effet, depuis au moins une cinquantaine d'années, l'école maternelle connaît une lente évolution marquée par un usage croissant de supports («fiches», affichages, étiquettes...) qui, s'ils mêlent différents types d'« écrits ", sont souvent composés de signes graphiques non linguistiques (Joigneaux, 2013a; Joigneaux, Laparra \& Margolinas, 2014). Peut-on pour autant parler de «nouvelle littératie scolaire» comme cela a pu être fait pour d'autres niveaux de la scolarité (Viriot-Goeldel \& Delarue-Breton, 2014), et ce faisant de «scolarisation » de l'école maternelle (Garnier, 2016) ? Tout dépend de la définition qu'on se donne de la littératie, et in fine de l'écrit. L'enjeu d'une réflexion un peu plus poussée que celles qui sont formalisées dans les programmes ou dans certaines recherches (Joigneaux, Laparra \& Margolinas, 2014) n'est donc pas que terminologique ou épistémologique. Selon en effet les spécifications de l'écrit, on n'aboutira pas aux mêmes constats.

2 Une telle réflexion est d'autant plus précieuse que même la littérature anglophone consacrée à la littératie, pourtant foisonnante et précoce relativement aux travaux francophones portant sur cet objet, n'aide pas à lever les ambiguïtés terminologiques qui l'entourent (Brockmeier \& Olson, 2009, p. 4-5 ; Joigneaux, 2013a). Selon les articles, les méthodologies ou les questions traitées, les conceptions - le plus souvent implicites - de la littératie sont très variables. Nous ferons l'hypothèse dans cet article qu'il en est ainsi précisément parce que la notion de littératie est rarement insuffisamment spécifiée, si bien qu'elle finit par renvoyer à des ensembles de phénomènes très fluctuants selon les usages qui en sont faits, comme peut en 
témoigner la croissance exponentielle de qualificatifs qui peuvent lui être accolés. Alors que pour une grande majorité de travaux ${ }^{1}$ elle reste très souvent associée aux compétences cognitives impliquées dans (l'apprentissage de) la lecture et l'écriture au sens le plus traditionnel de ces deux termes; des recherches plus récentes utilisent ce terme pour qualifier tous les modes de communication et de représentation dans lesquels intervient plus ou moins directement l'écrit, ce dernier terme pouvant alors même être utilisé dans l'acception élargie de traces graphiques (Chamberlin, 2002).

Étant donné que la plupart de ces travaux font référence à ceux de J. Goody et que ce dernier est un des chercheurs un des chercheurs qui s'est le plus évertué à spécifier ce qu'est et autorise ou facilite l'écrit, la mise en perspective de la littérature scientifique consacrée à la littératie qui va composer les deux premières parties de cet article s'appuiera de façon préférentielle sur un certain nombre de réflexions qu'il a développées, notamment dans un de ses ouvrages les plus cités, La Raison Graphique (Goody, 1978 [1977]). Dans la dernière partie, nous tenterons de montrer comment des approches à la fois "multimodales" et "reconnectées " de la littératie peuvent permettre de renouveler les éléments de réponses donnés aux questions que pose l'usage de l'expression de « littératie(s) scolaire(s)».

\section{La littératie, entre cognitivisme et culturalisme}

Depuis l'émergence des «New Literaties Studies » (Fraenkel \& Mbodj-Pouye, 2010), il est devenu courant, dans la littérature consacrée à la littératie, de distinguer deux principales approches, le plus souvent qualifiées respectivement de « cognitive » et de « socio-culturelle ». Outre son ancrage à la psychologie cognitive et aux méthodologies expérimentales, ce qui caractérise un peu plus la première approche est sa manière de délimiter ses objets de recherche. En effet, elle étudie avant tout comment des individus acquièrent et développent des compétences ou connaissances exclusivement cognitives et strictement délimitées (par exemple le développement de la conscience phonologique avant ou au cours de l'apprentissage de la lecture), le contexte social de ces processus étant au mieux relégué au statut de variable explicative (Cushman et al., 2001, p. 3).

Ce qui n'est pas sans liens avec le qualificatif «socio-culturel » et l'expression « New Literacy studies » (NLS par la suite) revendiqués généralement au sein des travaux composant la seconde approche. En effet, ces derniers s'intéressent davantage aux façons dont les usages de l'écrit et leur légitimité varient selon les contextes socioculturels, mais bien souvent sans trop s'attarder sur leurs caractéristiques cognitives. Ils ont aussi en commun de dénoncer le caractère normatif ou «idéologique " (Gee, 1992) de l'approche cognitive dans la mesure où en général les travaux la composant ne se préoccupent le plus souvent que du développement, chez l'enfant, des connaissances et compétences reconnues et valorisées par les institutions scolaires, et plus généralement occidentales, sans trop d'égards pour la multiplicité des littératies et des contextes sociaux plus «informels» dans lesquels se développent des pratiques de l'écrit plus «locales ", « situées", «intimes » ou "hétérodoxes » et de fait moins formalisées (Barton \& Hamilton, 1998 ; Barton, Hamilton, Ivanic, 2000). Mais si elles sont plus sensibles à l'ancrage socio-culturel des pratiques de l'écrit («literacy embedded »), ces recherches ont tendance à laisser à l'arrière-plan de leurs analyses, les spécificités cognitives de ces pratiques, de façon donc symétrique aux travaux 
composant l'approche cognitive. Ce que J. Gee (un des principaux protagonistes des NLS) par exemple a reconnu implicitement à l'issue de la controverse qu'il a entretenue avec $\mathrm{C}$. Snow, dans une suite d'articles où ces deux chercheurs ont cherché à clarifier les partis pris épistémologiques de ces deux approches : "NLS is interested not primarily, as is Professor Snow, in how cognitive changes within individual affect their nature of participation, but in how changes in the nature of participation affect cognition, socially situated identities, and the assessments made about individuals " (Gee, 2000, p. 126). Ce qui semble avant tout opposer ces deux approches de la littératie, ce ne serait donc pas leur ignorance ou la minoration de la dimension socio-culturelle ou cognitive des connaissances, compétences ou pratiques de l'écrit, comme leurs pourfendeurs respectifs se plaisent à l'écrire, mais plutôt l'importance relative qu'elles accordent à ces deux dimensions, et à travers elle, le sens de la causalité entre le social (ou la culture) et le cognitif (ou la pensée).

C'est sans doute aussi ce "grand débat ", aux solides ramifications philosophiques, qui sous-tend les controverses suscitées par les thèses de J. Goody sur les conséquences cognitives et sociales de l'apparition et de la diffusion de l'écrit (Olson \& Cole, 2006; Privat \& Kara, 2006). Ainsi, parce que J. Goody a tendance à ne considérer qu'un des sens de cette relation causale, c'est-à-dire les implications sociales et institutionnelles de l'invention et de la diffusion de l'écrit et des pouvoirs cognitifs qui peuvent lui être attribués, B. Street, l'un des fondateurs des NLS le qualifie de "cognitiviste» (Street, 1984, p. 10). Pourtant, bien qu'il ait moins travaillé l'autre sens de la causalité (la façon dont les pratiques socio-culturelles modulent la diffusion et les implications cognitives de l'écrit), il en fait parfois mention, surtout - il est vrai - dans des écrits postérieurs à La raison graphique. Comme par exemple dans Pouvoirs et savoirs de l'écrit: "Plus l'organisation de l'État et de l'économie devient complexe, plus la pression est grande pour que l'on aille en direction de la représentation graphique de la parole » (Goody, 2007 [2000], p. 49).

7 Une lecture attentive et surtout moins partiale du travail de J. Goody montre bien la difficulté à opposer de façon systématique les deux principales approches - cognitive ou socio-culturelle - de la littératie. Tous ses textes ou même certains de leurs extraits peuvent être situés en-deçà ou au-delà de la frontière qui est censée délimiter les contours de ces deux approches, selon le sens de la causalité privilégiée entre le social et le culturel. Cela montre bien, comme le constatent d'autres chercheurs, que le débat entre les tenants d'une approche « cognitive » et « socio-culturelle » de la littératie est loin d'être clos (Olson \& Torrance, 2009, xix) et que l'étude de la littératie reste « un chantier toujours ouvert » (Goody, 2006). Et ce débat doit se poursuivre, au risque sinon d'un durcissement des positions épistémologiques et théoriques de ces deux approches, ce qui serait dommage dans la mesure où elles nous semblent beaucoup plus complémentaires qu'exclusives (Purcell-Gates, Jacobson \& Degener, 2004), comme nous allons le montrer dans la suite de ce texte.

\section{Vers une approche multimodale et reconnectée de la littératie?}

8 Ainsi a-t-on pu reprocher à J. Goody son « cognitivisme » parce qu'il s'est avant tout intéressé aux "pouvoirs cognitifs» de l'écrit, sans se préoccuper des conditions sociales dans lesquelles ils peuvent ou non s'exercer. S'il n'a pas détaillé les pratiques 
culturelles qui permettent plus ou moins d'exploiter ce pouvoir, il en a ouvert la voie. D'abord parce qu'il remarque parfois - comme on l'a déjà souligné - que l'exploitation des pouvoirs cognitifs de l'écrit a été variable, bien que cumulative, selon les contextes socio-historiques. Ensuite, parce qu'il n'a eu de cesse de critiquer les schémas monocausaux de certains de ses devanciers, et plus particulièrement le déterminisme technologique qu'ils peuvent impliquer ${ }^{2}$. Enfin, il reste assez prudent dans ses formulations des relations causales pouvant exister entre l'écrit et ses implications cognitives : s'il s'intéresse aux pouvoirs cognitifs de l'écrit, il précise souvent que ce sont avant tout des pouvoirs facilitateurs d'opérations cognitives ${ }^{3}$.

9 Autrement dit, même si cela reste très théorique, J. Goody reconnaît parfois que toutes les pratiques culturelles de l'écrit n'ont pas les mêmes implications cognitives. Ce qui ouvre la voie à l'étude des façons dont diverses pratiques de l'écrit exploitent plus ou moins les pouvoirs facilitateurs d'opérations cognitives, selon notamment les contextes socio-historiques dans lesquelles elles sont (ont été) déployées. Cette dernière inférence est une des façons de "reconnecter" (ibid., p. 72) les approches cognitives et socioculturelles de la littératie ${ }^{4}$. Elle permettrait en particulier d'éviter le caractère très descriptif et relativiste de certains travaux de l'approche socio-culturelle quand ils ne spécifient la diversité des littératies qu'ils étudient que par les spécificités des contextes dans lesquelles elles sont observées - les littératies "locales»-, en sousestimant de ce fait ce qu'elles peuvent avoir en commun (ibid., p.18). Et cela permettrait aussi de comprendre autrement les sources socio-culturelles des inégalités scolaires: selon les régularités des usages de l'écrit des élèves - leurs habitus littératiens (Privat, 2006) - certains de ses pouvoirs facilitateurs ne sont pas exploités de la même façon ni dans la même mesure.

10 L'amplification d'un tel mouvement de « reconnection » est d'autant plus probable qu'il déborde le champ de la littérature scientifique consacrée à la littératie dans la mesure où il semble aussi travailler les recherches dans le domaine de l'anthropologie du langage, comme l'a bien relevé en particulier W. Hanks (1996). Suite à une mise en perspective des travaux de ce champ de recherches, qui est traversé lui aussi par des approches plus ou moins socio-culturellement contextualisées, ce dernier a ainsi mis en évidence que délaisser les apports des approches les moins contextualisées qu'il qualifie de «formalistes », celles qui étudient les spécificités sémiotiques des ressources langagières indépendamment des contextes de leurs usages, conduit généralement au biais inverse, c'est-à-dire à la sous-spécification des ressources propres aux différents systèmes sémiotiques impliqués dans les pratiques socio-culturelles étudiées (ibid., p. 1-15). C'est la raison pour laquelle il cherche lui aussi à intégrer dans son champ de recherche les apports des approches les plus formalistes et contextuelles du langage.

Dans la perspective ouverte par Hanks, cette troisième approche se caractérise aussi par une mise en question du champ de pertinence des usages du terme de langage et de ses déclinaisons orales et écrites. En particulier, sont discutées la frontière entre langage corporel et langage oral ainsi que la nature des relations entre ce qui est oralisé et ce qui est écrit. Là encore, le parallèle peut être fait entre les nouvelles orientations de l'anthropologie du langage et celles du champ des recherches axées sur la littératie. En effet, depuis le début des années 2000, les relations entre les différentes ressources sémiotiques sur lesquelles peuvent s'appuyer différents usages de l'écrit sont étudiées plus systématiquement au sein d'une nouvelle approche de la littératie qui est souvent qualifiée de multimodale (Jewitt \& Kress, 2003). Il s'agit dans ce cadre d'étudier 
comment se construit la compréhension au cours d'interactions entre différents individus qui utilisent pour ce faire non seulement la parole ou l'écrit, mais aussi beaucoup d'autres modes de communication et de représentation, parmi lesquels les images, les regards, les gestes, les mouvements, la musique, les effets sonores... (ibid., p. 1).

12 Les approches multimodales et reconnectées à la façon de D. R. Olson ont le mérite de reposer la question des frontières et de la nature de l'écrit, et plus particulièrement des relations qu'elle peut entretenir avec la parole et plus généralement l'oral. En effet, elles ont en commun de rejeter une conception de la littératie restreinte aux systèmes langagiers de nature alphabétique, largement présente dans la littérature consacrée à cet objet. Dans l'acception "phonocentrée » (Derrida, 1967, p. 23) encore dominante, l'écrit est considéré comme une simple transcription graphique de la parole et non comme un langage à part entière (Harris, 1995, p. 3). Ce qui n'est pas le cas de ces travaux dont un autre trait distinctif est donc de partager (plus ou moins explicitement) une conception davantage extensive de la littératie (Joigneaux, Laparra \& Margolinas, 2014, p. 4). En effet, à la suite notamment de certains travaux de J. Goody, ces travaux-ci incluent dans la littératie toutes «les formes graphiques que le plus souvent on ne prononce pas ou qui sont imprononçables » (Goody, 1978 [1977], p. 198), tels les plans, les schémas, les diagrammes, les tableaux, les listes, la mise en forme graphique des syllogismes, les chiffres, les typographies, les mises en page ou en livre, les signes de ponctuation, (et parfois) les représentations iconiques...

13 Mais par rapport aux perspectives ouvertes par W. Hanks ou par D. R. Olson, les spécificités sémiotiques des différents modes de représentation et de communication recensés au sein de l'approche multimodale, en particulier celles de l'écrit, sont beaucoup moins travaillées si bien que ce qui distingue les littératies multimodales entre elles semble être avant tout et presque exclusivement la pondération et l'articulation de ces différents modes. La multiplication des qualificatifs sensoriels et/ ou sémiotiques (littératie visuelle, iconique...) qui sont aujourd'hui associés au terme de littératie constitue un indice de cette façon de considérer la multimodalité en son sein. On semble s'être éloigné de l'approche "reconnectée » telle qu'elle est envisagée par D. R. Olson notamment à partir des travaux de J. Goody, autrement dit la caractérisation des pratiques socio-culturelles de l'écrit en fonction de leurs manières de plus ou moins exploiter les pouvoirs de l'écrit et de faciliter en conséquence certaines opérations cognitives.

Cela peut s'expliquer par les difficultés conceptuelles qui peuvent alors être rencontrées. En effet, si J. Goody a cherché à systématiser les façons dont certaines opérations cognitives pouvaient être facilitées par les inventions et la diffusion des systèmes écrits alphabétiques, puis plus largement par un certain nombre de dispositifs graphiques qui ne se prononcent pas, il s'est beaucoup moins intéressé à leurs implications sur les pratiques orales, comme il le reconnaît explicitement à la fin de $L a$ raison graphique: "J'ai conscience d'avoir eu trop tendance, dans les pages qui précèdent, à me laisser enfermer dans une autre dichotomie en opposant l'énoncé verbal au texte, l'oral à l'écrit » (ibid., p. 252). En outre, le foisonnement de la pensée de J.Goody sur les pouvoirs facilitants de l'écrit sur le plan cognitif est tel que les synthèses qu'il propose sur le sujet semblent souvent parcellaires, si bien qu'il est assez difficile d'avoir une appréhension globale et unifiée de toutes opérations intellectuelles que l'écrit peut rendre possibles ou plus aisées et des relations qu'elles entretiennent avec toutes les 
caractéristiques sémiotiques de l'écrit. Par exemple, dans le passage suivant, Goody distingue "deux fonctions principales de l'écriture " qu'il met en relation avec un certain nombre d'opérations intellectuelles qu'elles peuvent faciliter: "L'écriture a deux fonctions principales. L'une est le stockage de l'information, qui permet de communiquer à travers le temps et l'espace et qui fournit à l'homme un procédé de marquage, de mémorisation et d'enregistrement. [...] la seconde fonction, celle qu'a l'écriture en assurant le passage du domaine auditif au domaine visuel, ce qui rend possible d'examiner autrement, de réarranger, de rectifier des phrases et même des mots isolés ». (ibid., p. 145). Mais dans d'autres passages, notamment à propos des tableaux ou des listes, il généralise les implications cognitives du caractère visuel des représentations graphiques: "Le tableau établit une relation entre les articles d'une même ligne aussi bien qu'entre ceux d'une même colonne » (ibid., p. 213). En effet, il n'est plus question ici d'unités linguistiques de différents ordres, mais d' "articles », ce qui est cohérent avec son insistance dans cet ouvrage à montrer que ce sont les systèmes d'écriture en général, et pas seulement alphabétiques, qui facilitent la mise en relation et la catégorisation d'éléments linguistiques ou non (des "articles») parce qu'ils permettent de sortir ces derniers de leurs contextes d'usage initiaux (" décontextualisation ») et ainsi de les rapprocher visuellement au sein d'un contexte exclusivement graphique (« recontextualisation »). Abordées par J. Goody dans d'autres passages de la Raison Graphique, ces dernières opérations intellectuelles semblent relever d'un ordre de généralité supérieur en termes d'implications cognitives que simplement "réarranger, de rectifier des phrases et même des mots isolés ", même si elles découlent elles aussi du caractère visuel des représentations graphiques. Dernière remarque sur cette difficulté d'avoir une vision unifiée et systématisée des pouvoirs facilitateurs de l'écrit: la fonction facilitatrice de stockage de l'information ne serait pas possible sans l'existence de la fonction de l'écriture d'assurer le passage du domaine auditif au domaine visuel, comme le soutient J. Goody dans d'autres passages : "Non seulement on donne à l'information, en la mettant par écrit, à la fois une plus grande fixité et plus grande souplesse d'utilisation (elle peut être réordonnée de diverses manières), mais on s'assure aussi un moyen de la stocker» (ibid., p. 159). En conséquence, relèvent-elles bien du même ordre de généralité ?

\section{Quelques exemples d'opérationnalisation d'une approche à la fois reconnectée et multimodale de la littératie}

15 Mais encore une fois, les développements de J. Goody sur les implications cognitives potentielles de l'usage de l'écrit restent incontournables, malgré leur foisonnement, dès lors qu'on s'intéresse davantage à ce que des littératies peuvent avoir en commun et moins à leur pluralité ou à leur localité en soi. Car nous avons vu que le cadre théorique de J. Goody permet d'établir des ponts entre trois des principales approches de la littératie (cognitive, socio-culturelle et multimodale) et ainsi ouvrir la voie à une approche reconnectée dans le sens où elle permettrait d'intégrer un certain nombre de leurs apports respectifs. En outre, c'est sans doute l'approche la plus prometteuse pour mieux répondre aux questions que nous avons posées au début de ce texte. En effet, il nous semble que dès que l'on s'intéresse à la construction des inégalités socio-scolaires et que l'on cherche en conséquence à mieux identifier ce qui est le plus généralement 
attendu à l'école en termes d'usages de l'écrit et comment les élèves dans leur diversité sur ce plan y répondent plus ou moins - un des principaux facteurs de réussite ou d'échec scolaire (Lahire, 1993 ; Laparra, 2005, p. 44 ; Laparra, 2006, p. 244) -, il devient nécessaire d'étudier comment certains pouvoirs facilitateurs de l'écrit (ses potentialités sémiotiques) sur le plan cognitif sont diversement exploités selon les contextes sociaux et/ou les prédispositions sur ce plan des élèves. C'est ce que nous allons entreprendre à l'aide de deux exemples d'analyses réalisées à partir de ce schème d'interprétation et qui portent sur les pratiques littératiennes d'enfants fréquentant l'école maternelle.

La première étudie comment des élèves de Grande Section de maternelle exploitent plus ou moins la possibilité d'avoir une vision d'ensemble des traces graphiques (non alphabétiques) qu'ont laissées leurs opérations intellectuelles passées pour en contrôler la cohérence et planifier en conséquence celles qui vont suivre (Joigneaux \& Rochex, 2008 ; Joigneaux, 2009). Nous avons pour cela montré que les élèves qui font le moins d'erreurs au cours des activités réalisées sur des «fiches » (feuilles pré-imprimées qu'ils peuvent compléter), quelle que soit leur nature, sont ceux qui le plus régulièrement prennent le temps de suspendre l'enchainement de leurs tracés graphiques pour avoir une vision d'ensemble de l'espace graphique sur lequel ils travaillent : durant ces moments, ils ont tendance à pointer les différents éléments graphiques (leurs traces ou ceux qui sont pré-imprimés) qu'ils doivent prendre en considération, y compris donc ceux qu'ils ont eux-mêmes produits; parfois aussi ils accroissent la distance qui les sépare de leur espace de travail comme pour en avoir une meilleure vision d'ensemble; enfin, il leur arrive alors de rapprocher, cette fois matériellement, des éléments graphiques tels des modèles ou des supports graphiques contenant des informations importantes car nécessaires à la réalisation de l'activité proposée. Tous ces petits gestes d'étude ont en commun d'exploiter des ressources sémiotiques de l'écrit décrites par J. Goody, notamment la possibilité qu'elles offrent de rapprocher au sein d'un même espace graphique des éléments tirés de contextes spatio-temporels qui peuvent être très éloignés les uns des autres, donc de les recontextualiser pour qu'ils puissent être reconsidérés visuellement et de façon presque instantanée (Goody, 1978 [1977], p. 87, 145, 216 ; Harris, 1995, p. 38). C'est ce genre de potentialité sémiotique qu'exploitent les différents types de rapprochements corporels et matériels (rapprochement de supports, des traces de leurs activités passées avec celles qui sont à venir, d'autres éléments graphiques...) réalisés par les élèves dont les pratiques sont ici analysées. Mais tous les élèves ne sont pas aussi (pré-)disposés à faire usage de ces micro-pouvoirs de l'écrit. Comme le révèlent non seulement leurs erreurs - ces erreurs ont tendance à s'enchainer de façon cohérente - mais aussi leurs conduites corporelles et temporelles, la plupart des élèves issus des milieux sociaux les plus défavorisés " circulent » beaucoup moins dans les espaces graphiques qu'ils ont à leur disposition, et leurs prises (visuelles) d'informations sont donc beaucoup plus ponctuelles et parcellaires.

17 Le second exemple d'analyse porte sur la comparaison de pratiques de "lectures partagées " d'albums de jeunesse réalisées chez eux par un échantillon d'enfants et leurs parents, ces derniers ayant des niveaux d'études très contrastés (Bonnéry \& Joigneaux, 2015). Là encore, ce sont les parents qui ont les diplômes les plus élevés qui font le plus de rapprochements entre des éléments lus à différents moments de la lecture ou qui peuvent être mobilisés pour en interpréter les contenus. Plus que les autres, ils interrompent la lecture des textes pour poser des questions à leur propos et 
souvent pour mettre en relation ce qui est en train d'être lu et ce qui l'a été ou les illustrations des albums, ce qui les conduit parfois à revenir en arrière (revenir aux premières pages lues) pour pointer des éléments iconiques à l'appui de ce type de questions. Comme ces gestes d'étude sont très proches de ceux qui ont été saisis à l'issue de l'analyse des observations des usages de l'écrit des élèves qui réussissent le mieux en maternelle, on peut penser qu'ils constituent le répertoire des premiers usages de l'écrit les plus valorisés à l'école, qui pour cette raison peut être qualifié de littératie scolaire et qui pourrait constituer une des faces des habitus littératiens les plus valorisées dès l'école maternelle, mais les plus cachées aux approches exclusivement cognitives.

\section{Conclusion}

18 Parce qu'elle est traversée par des approches qui dialoguent peu, la littérature scientifique qui s'est développée autour de la notion de littératie est encore «en chantier ». Comme, de ce fait, la notion elle-même. Ainsi sa délimitation conceptuelle reste variable, si bien que le choix de l'utiliser au singulier ou au pluriel reste discutable et discuté. Mais notre analyse de ce qui caractérise et parfois oppose les approches cognitives et socioculturelles plaide pour une (ré)ouverture de ce chantier, à partir notamment des nombreuses relectures qui peuvent être faites du travail séminal de J. Goody. Car ces approches nous semblent plus complémentaires qu'orthogonales. Il ressort en effet du débat serré entre C. Snow et J.P. Gee - deux des principales figures de l'approche cognitive et socio-culturelle, respectivement - qui a eu lieu au début des années 2000, que ce qui les distingue ne sont pas tant les méthodologies qu'elles privilégient (expériences et tests d'un côté, ethnographie de l'autre) que le sens de la relation causale étudiée entre d'une part les pratiques socio-culturelles de l'écrit, et d'autre part, les connaissances ou compétences cognitives relatives à l'écrit. Alors que l'approche socio-culturelle, dans une perspective très vygotskienne, a tendance à supposer que ce sont les pratiques de l'écrit et leurs contextes qui développent cet ensemble de compétences ou de connaissances, l'approche cognitive fait - souvent implicitement) - l'hypothèse inverse.

19 Nous avons essayé de montrer dans cet article, quelques exemples d'analyses empiriques à l'appui, qu'une troisième voie était envisageable en reconnectant ces deux principales approches de la littératie en s'appuyant notamment sur les travaux de J. Goody. En effet l'ancrage anthropologique de ces derniers est compatible avec une approche qui privilégie l'étude des pratiques culturelles (dans toute leur diversité) alors que son "cognitivisme" supposé peut conduire à mieux spécifier ce qu'elles partagent ou non sur le plan cognitif, sans pour autant que ce dernier se limite à l'ensemble de compétences ou de connaissances habituellement étudiées dans le cadre de la psychologie cognitive.

Ce cadre d'analyse est tout aussi compatible avec les travaux qui s'intéressent à la multimodalité de la littératie: dès lors qu'on adopte un grain fin d'observations et d'analyses des pratiques de l'écrit, on ne peut qu'être "saisi " par la façon dont ces dernières varient selon les modes de communication et de représentation auxquels elles sont articulées. Mais par rapport à la plupart des travaux étudiant cette multimodalité, la spécification de la diversité relative des usages de l'écrit ne se limite pas à l'établissement de configurations multimodales. Sur ce plan aussi, la façon de 
procéder de J.Goody nous semble précieuse. Même si - surtout - dans La raison graphique, ce dernier ne s'est que très sporadiquement intéressé aux façons dont les modes oraux et écrits de communication pouvaient diversement s'articuler, il n'en a pas moins développé une réflexion soutenue sur les opérations intellectuelles que ces deux modes permettent plus ou moins. Ce qui ouvre la voie à l'étude des implications cognitives des différences configurations possibles entre les modes écrits et oraux de communication et de représentation, voie qui commence à être explorée par les derniers travaux de Claire Margolinas et Marceline Laparra (Laparra \& Margolinas, 2016).

\section{BIBLIOGRAPHIE}

BARTON, D. \& HAMILTON, M. (1998). Local literacies. Reading and writing in one community. Londres/ New York : Routledge.

BARTON, D., HAMILTON, M. \& IVANIČ, R. (éds.) (2000). Situated Literacies. Reading and writing in context. Londres/New York : Routledge.

BONNÉRY, S. \& JOIGNEAUX, C. (2015). « Des littératies familiales inégalement rentables scolairement ». Le français aujourd'hui 190, p. 23-34.

BRockMEIER, J. \& OlSON, D. R. (2009). « The literacy episteme ». In : Olson, D. R. \& Torrance, N. (éds). The Cambridge Handbook of Literacy. Cambridge/New York : Cambridge University Press, p. 3-21.

CHAMBERLIN, E. (2002). « Hunting, tracking and reading. In : Brockmeier, J., Wang, M. \& Olson, D. R. (éds.), Literacy, narrative and culture. Londres/New York : Routledge, p. 67-85.

CUSHMAN, E. et al. (2001). Literacy: A critical sourcebook. Boston : Bedford/St. Martin's. DERRIDA, J. (1967). De la grammatologie. Paris : Éditions de Minuit.

FINNEGAN, R. (2006). « Not by words alone : Reclothing the "oral” ». In : Olson, D. R. \& Cole, M. (éds.). Technology, literacy, and the evolution of society. Implications of the work of Jack Goody. Mahwah/ Londres : Lawrence Erlbaum, p. 265-287.

FRAENKEL, B. \& MBODJ-POUYE, A. (2010). « Introduction. Les New Literacy studies, jalons historiques et perspectives actuelles ». Langage et société 133, p. 7-24.

GARNIER, P. (2016). Sociologie de l'école maternelle. Paris : Presses universitaires de France.

GEE, J. P. (1992). The social mind. Language, ideology, and social practice. New York : Bergin \& Garvey.

GEE, J. P. (2000). « The Limits of Reframing: A Response to Professor Snow ». Journal of Literacy

Research 32 (1), p. 121-128.

GOODY, J., (1978) [1977]. La raison graphique. La domestication de la pensée sauvage. Trad. de l'anglais par J. Bazin \& A. Bensa. Paris, Éditions de Minuit.

GOODY, J. (2006). « La littératie, un chantier toujours ouvert. Entretien avec Jack Goody ». Trad. de l'anglais par K. Birat. Pratiques 131-132, p. 69-75. 
Goody, J., (2007) [2000], Pouvoirs et savoirs de l'écrit. Trad. de l'anglais par C. Maniez. Paris : La Dispute.

Goody, J. \& Watt, I. (2006) « Les conséquences de la littératie ». Trad. de l'anglais par J.-C. Lejosne. Pratiques 131-132, p. 31-68

HANKS, W. F. (1996). Language \& communicative practices. Boulder : Westview Press.

HARRIS, R. (1995). Signs of writing. Londres/New York : Routledge.

JEWITT, C. \& KRESS, G. R. (éds.) (2003). Multimodal literacy. New York : Peter Lang.

JOIGNEAUX, C. (2009). « La construction de l'inégalité scolaire dès l'école maternelle ». Revue française de pédagogie. Recherches en éducation 169, p. 17-28.

JoIGNEAUX, C., (2013a). « La littératie précoce. Ce que les enfants font avec l'écrit avant qu'il ne leur soit enseigné ». Revue française de pédagogie. Recherches en éducation 185, p. 117-162.

JoIGNEAUX, C. (2013b). « Littératie, forme et inégalités scolaires : le cas de la "scolarisation" de l'école maternelle », Le Français aujourd'hui 183, p. 41-50.

JOIGNEAUX, C. (2015). «La diffusion des fiches à l'école maternelle », Spirale. Revue de recherches en éducation 55, p. 57-65.

JoIGNEAUX, C., LAPARRA, M., \& MARGolinAS, C. (2014). « Une dimension cachée du curriculum réel de l'école maternelle : la littératie émergente?». Actes du colloque Sociologie et didactiques : vers une transgression des frontières ?. 13-14 septembre 2012. Lausanne : Haute école pédagogique de Lausanne. En ligne : http://www.hepl.ch/files/live/sites/systemsite/files/uer-agirs/actualites/ colloque-didactiques-et-sociologie/actes-colloque-sociologie-et-didactiques-philippe-losego-ueragirs-hep-vaud.pdf.

JOIGNEAUX, C. \& ROCHEX, J.-Y., (2008) « La construction de l'élève à l'école maternelle : regards croisés et apports de Vygotski, Bernstein et Goody ». In : Brossard, M. \& Fijalkow, J. (dirs). Vygotski et les recherches en éducation et en didactiques. Pessac : Presses universitaire de Bordeaux, p. 73-87.

LAHIRE, B. (1993). Culture écrite et inégalités scolaires. Sociologie de «l'échec scolaire »à l'école primaire. Lyon : Presses universitaires de Lyon.

LAPARRA, M. (2005). « L'écrit en maternelle : bricolage ou opération cognitive ? ». In : Ramognino N. \& Vergès P. (éds.). Le français hier et aujourd'hui. Politique de la langue et apprentissages scolaires. Études offertes à Viviane Isambert-Jamati. Aix-en-Provence : Publications de l'université de Provence, p. 39-47.

LAPARRA, M. (2006). « La Grande Section de maternelle et la "Raison Graphique" ». Pratiques 131-132, p. 237-249.

LAPARRA, M., MARGOLINAS, C. (2016). Les premiers apprentissages scolaires à la loupe. Des liens entre énumération, oralité et littératie. Louvain-la-Neuve : De Boeck.

Ministère de l'Éducation nationale et de la Jeunesse (2015). « Programme d'enseignement de l'école maternelle ». Bulletin officiel spécial n² 2 du 26 mars 2015.

OLSON, D. R. \& COLE, M. (éds) (2006). Technology, literacy, and the evolution of society. Implications of the work of Jack Goody. Mahwah/Londres : Lawrence Erlbaum.

OlSON, D. R., TORRANCE, N. (éds) (2009). « Preface ». In : Olson, D. R., Torrance, N. The Cambridge Handbook of Literacy. Cambridge/New York : Cambridge University Press, pp. xiii-xxi. 
PRIVAT, J.-M. (2006). « Un habitus littératien? ». Pratiques 131-132, p. 125-130.

PRIVAT, J.-M. \& KARA, M. (éds) (2006). « La littératie. Autour de Jack Goody », Pratiques 131-132.

PURCell-gates, V., JACoBson, E. \& Degener, S. (2004). Print Literacy Development. Uniting Cognitive and Social Practice Theories, Cambridge : Harvard University Press.

STREET, B. V. (1984). Literacy in theory and practice. Cambridge : Cambridge University Press.

VIRIOT-GOELDEL, C. \& DELARUE-BRETON, C. (2014). « Des textes composites à l'école : nouvelle littéracie scolaire, apprentissages et inégalités ». Spirale. Revue de recherches en éducation, 53, p. 21-31.

\section{NOTES}

1. Pour préparer la rédaction d'une note de synthèse consacrée à la littératie précoce (ou émergente), nous avons référencé plus de 1500 travaux portant sur la littératie (Joigneaux, 2013a).

2. Par exemple il écrit dans la Raison Graphique: "Je n'entends nullement par là privilégier une séquence causale relevant d'un déterminisme technologique [...] une explication unilinéaire et unicausale [...]. Il y a une voie médiane entre choisir une cause unique et rejeter toute explication causale, entre noyer tout dans la causalité structurale et la cohérence fonctionnelle et isoler un unique facteur matériel comme cause dominante ou même déterminante; reste ouvert tout champ des faisceaux de causes convergentes, des mécanismes d'action en retour " (Goody, 1978 [1977]), p. 49). Et à d'autres endroits de cet ouvrage, J. Goody remarque (ce sont - il est vrai - de simples remarques) que les usages de la « raison graphique » ont socio-historiquement varié (ibid., p. 78-83, 100, 107).

3. Dès la Raison Graphique J. Goody a tendance à privilégier les verbes «faciliter » et «permettre » pour traduire les implications cognitives l'écrit. À cet égard, R. Finnegan remarque que dans ses premiers écrits sur la question, notamment dans l'article écrit avec I. Watt en 1963 (Goody \& Watt, 2006), Goody employait l'expression " conséquences de l'écrit », puis progressivement, il a privilégié le terme d'implications (Finnegan, 2006, p. 269).

4. Sans toutefois faire une telle référence aux pouvoirs facilitateurs de l'écrit objectivés par J. Goody (à l'exception de D. R Olson), quelques recherches ont déjà exploré cette « troisième voie » (notamment Jewitt \& Kress, 2003 ; Purcell-Gates, Jacobson \& Degener, 2004 ; Brockmeier \& Olson, 2009).

\section{RÉSUMÉS}

Parce qu'elle est traversée par des approches qui dialoguent peu, la littérature scientifique qui s'est développée autour de la notion de littératie est encore «en chantier ». Comme, de ce fait, cette notion elle-même. L'analyse de ce qui caractérise les approches cognitives et 
socioculturelles proposée dans cet article, plaide pour une (ré)ouverture de ce chantier, à partir notamment des travaux de J. Goody. En effet leur ancrage à la fois anthropologique et cognitif peut servir de socle théorique à une approche «reconnectée » de la littératie, une approche permettant de mieux intégrer à l'étude des variations socio-culturelles et multimodales des pratiques de l'écrit leurs spécificités cognitives, par exemple en montrant comment ces variations sont liées aux différentes manières d'exploiter les "pouvoirs cognitifs" de l'écrit. Dans la deuxième partie de l'article, nous cherchons à montrer l'intérêt de cette approche reconnectée pour mieux cerner la littératie scolaire et son implication dans la reproduction des inégalités socio-scolaires.

Because it is crossed by approaches that have little dialogue, the scientific literature that has developed around the concept of literacy is still « in progress ». As it is for this this concept itself. The analysis, proposed in this article, of what characterizes the cognitive and sociocultural approaches argues in favor of a (re)opening of this work site, particularly from J. Goody's work. Indeed, their integration both anthropologic and cognitive can serve as a theoretical basis for a « reconnected » approach to literacy, an approach to better integrate the study of socio-cultural and multimodal variations of written practices into their cognitive specificities, for instance by showing how these variations are related to different ways of using the « cognitive powers " of writing. In the second part of the article, we seek to demonstrate the interest of this reconnected approach in order to better understand the school literacy and its implication in the reproduction of socio-school inequalities.

\section{INDEX}

Mots-clés : littératie, cognitif, culture, école, multimodalité

Keywords : literacy, cognitive, culture, school, multimodality

\section{AUTEUR \\ CHRISTOPHE JOIGNEAUX}

Université Paris 8 Vincennes - Saint-Denis, Circeft-Escol, F-93526 Saint-Denis, France 